\title{
Comparison of Intubation Characteristics of McCoy Laryngoscope and LMA CTrach with Cervical Collar in Situ- A Simulation Study
}

\author{
Pawan Deshmukh ${ }^{1}$, Dnyanada Deshmukh², Manisha Bhatt Dwivedi ${ }^{3}$ \\ ${ }^{1}$ Department of Anaesthesia, SSIMS, Bhilai, Chhattisgarh, India. ${ }^{2}$ Department of Anaesthesia, SSIMS, Bhilai, \\ Chhattisgarh, India. ${ }^{3}$ Department of Anaesthesia, SSIMS, Bhilai, Chhattisgarh, India.
}

\section{ABSTRACT}

\section{BACKGROUND}

Cervical spine motion restriction is an integral component of protocol for management of trauma victims. The use of rigid cervical collar for the same, presents a hurdle in airway management in patients where intubation is mandated for successful resuscitation. Hence, techniques alternative to conventional laryngoscopy need to be explored through simulation studies, to ease the process of intubation and benefit the actual trauma victims. We wanted to assess the performance of McCoy laryngoscope and LMA CTrach assembly and compare the intubation characteristics in patients with cervical collar.

\section{METHODS}

80 patients of ASA status I or II, scheduled for elective surgery requiring general anaesthesia and endotracheal intubation were randomly allocated to two groups- A and B. Patients in Group A were intubated using McCoy laryngoscope and Group B using LMA CTrach, with cervical collar in situ. Airway assessment included measuring thyromental distance, observing MPC grade and measuring inter-incisor distance, before and after application of semirigid cervical collar. Glottic view was noted using modified Cormack-Lehane grading. Device insertion time, total intubation time, number of attempts, haemodynamic factors and airway complications during the procedure were noted.

\section{RESULTS}

There was decrease in inter-incisor distance and worsening of MPC grade in both groups post application of cervical collar. The time taken for device insertion in Group A was $16.95+3 \mathrm{sec}$, and in Group B was $33+4 \sec (\mathrm{P}=0.0001)$. The total intubation time in Group A was $40.4+6 \mathrm{sec}$ and in Group B was $57.4+4.37 \sec (\mathrm{P}=$ 0.0001). CL grade I was more common in Group B (31) than Group A (17) (P= 0.003). The number of attempts required, mean haemodynamic parameters and airway complication were comparable between the two groups.

\section{CONCLUSIONS}

The McCoy laryngoscope requires less time to obtain glottic view and subsequent intubation, but LMA CTrach provides better glottic exposure. Thus, LMA CTrach has better performance characteristics in patients with cervical collar in situ.
Corresponding Author: Dr. Dnyanada Deshmukh Flat No. 602, Faculty Quarters, SSIMS, Junwani, Bhilai-490020, Chhattisgarh, India.

E-mail: dnyanada.shinde@gmail.com

DOI: 10.14260/jemds/2020/337

Financial or Other Competing Interests: None.

How to Cite This Article:

Deshmukh P, Deshmukh D, Dwivedi MB. Comparison of intubation characteristics of McCoy laryngoscope and LMA CTrach with cervical collar in situ- a simulation study. J. Evolution Med. Dent. Sci. 2020;9(19):1544-1548, 10.14260/jemds/2020/337

Submission 12-03-2020,

Peer Review 24-04-2020,

Acceptance 30-04-2020,

Published 11-05-2020.

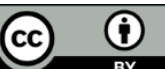

\section{KEY WORDS}

Cervical Collar, McCoy Laryngoscope, LMA CTrach, Simulation 


\section{BACKGROUND}

The incidence of cervical spine injury has been reported to be $1-4 \%$ in all major trauma victims and $4 \%$ to $8 \%$ in patients with head injury.1,2 Airway management requiring orotracheal intubation is an important key to successful resuscitation of trauma victims. Failure to immobilize cervical spine during the process can result in devastating neurologic outcomes. ${ }^{3}$ Thus, ATLS guidelines suggests the use of cervical collar for cervical spine motion restriction in trauma patients. ${ }^{4}$

The use of cervical collar has been shown to decrease the inter-incisor distance and increase the incidence of CormackLehane grades 3 and 4 laryngoscopic views (up to 64\%). ${ }^{5}$ Thus the scope of using conventional laryngoscopy for intubation is limited, as the collar may restrict the range of neck movement necessary for optimal alignment of the oral, pharyngeal and laryngeal axes for exposure of vocal cords ${ }^{6}$. Higher failure rates of tracheal intubation have prompted efforts to search for alternatives to improve the success rate of intubation.

Although fibreoptic bronchoscopy is considered gold standard in such scenarios, ${ }^{7}$ its use is limited by the need for expertise, availability, need for patient cooperation and presence of blood or secretions, which may hamper the view. In the present era, video laryngoscopes have been increasingly used in difficult intubation scenarios. The LMA CTrach system for airway management and endotracheal intubation is based on the Intubating Laryngeal Mask Airway (LMA Fastrach) system. ${ }^{8}$ The LMA CTrach consists of an airway with integrated fiberoptic channels, and a detachable LCD screen which enables the process of endotracheal intubation through laryngeal mask airway and is indicated as a device for achieving and maintaining airway in both anticipated and unexpected difficult intubation situations. Similarly, McCoy laryngoscope can convert a CormackLehane grade 2 or 3 to grade 1 or 2 at intubation and may help laryngeal visualization in patients with limited neck extension. ${ }^{9,10}$ Moreover, it is easily available and does not require much expertise to operate. In this randomised control study, we have compared the intubation characteristics of these two devices, in patients with cervical collar simulating cervical spine injury. As yet, there have been no direct comparison studies between McCoy laryngoscope and LMA CTrach in this scenario.

\section{METHODS}

The present randomized control study was conducted after obtaining clearance from the hospital Ethical Committee and Scientific Committee. A total of 80 patients of ASA status I or II, within age group of 18-50 years, scheduled for elective surgery requiring general anaesthesia and endotracheal intubation were selected. A written consent was taken after completely explaining the procedure to the patients. They were randomized using online randomization technique (http://www.randomization.com).

Patients with anticipated difficult airway (inter-incisor distance $<4 \mathrm{~cm}$, Mallampati grade III and IV, thyromental distance $<6 \mathrm{~cm}$ ), obesity (body mass index $>30 \mathrm{Kg} \cdot \mathrm{m}^{-2}$ ), severe respiratory or cardiac disease, cervical spine pathology and temporo-mandibular joint dysfunction were excluded from the study. All the patients underwent airway assessment and the following parameters were notedthyromental distance (TMD), inter-incisor distance (IID) and Mallampati class (MPC grade) with and without cervical collar (annexure 1), by anaesthesiologist blinded to group allocation. In Group A the patients were intubated by direct laryngoscopy technique using McCoy laryngoscope and those in Group B were intubated using LMA CTrach, with cervical collar in situ in both the groups.

All patients were kept nil per oral for minimum 8 hours prior to surgery. On arrival to the operation theatre, baseline parameters i.e. mean arterial pressure (MAP), heart rate (HR) and $\mathrm{SpO}_{2}$ were recorded. Appropriately sized rigid Philadelphia cervical collar was fitted as per manufacturer's instructions. All patients underwent standard anaesthesia induction with Inj. Glycopyrrolate $4 \mu \mathrm{g} / \mathrm{kg}$ and Inj. Fentanyl 2 $\mu \mathrm{g} / \mathrm{kg}$ I.V. as premedication and induction with Inj. Propofol 2 $\mathrm{mg} / \mathrm{kg}$. After confirming adequate mask ventilation with Sevoflurane $2 \%$ in Oxygen $10 \mathrm{~L} / \mathrm{min}$, Inj. Vecuronium bromide $0.1 \mathrm{mg} / \mathrm{kg}$ I.V. was administered. Intubation was attempted after $3 \mathrm{~min}$ to obtain optimal intubating conditions. Intubations were performed by senior anaesthetist with at least $2 y r s$ experience and adequate experience of more than 40 intubations in both techniques. For both the devices, the appropriate size and technique was used according to the manufacturer's guidelines ${ }^{8}$. A specially developed; straight, cuffed, silicone, wire-reinforced, reusable tracheal tube was used for the CTrach group patients and a standard curved polyvinylchloride tube for the McCoy group patients. The same haemodynamic parameters were recorded at appropriate intervals.

Device insertion time (DIT) was noted as the time between handling the device and obtaining a view of the glottis. Best view of the glottis was noted according to the modified Cormack-Lehane grading (annexure 1). Total intubation time (TIT) was noted as the time between handling the device and successful ventilation via the tracheal tube indicated by appearance of $\mathrm{EtCO}_{2}$ waveform. The intubation technique was considered as failed if tracheal intubation was not achieved with maximum 3 attempts. Then intubation was attempted by standard laryngoscopy technique after removal of cervical collar. Patients with failed intubation were excluded from the study. It was not possible to blind the anaesthetist performing the procedure for obvious reasons.

\section{Statistical Analysis}

Allowing for an $\alpha$ error of 0.05 and $\beta$ error of 0.2 (power of $80 \%)$, to detect a difference of 20 seconds for the total intubation time with a standard deviation of 30 seconds, a sample size of 35.28 for each Group was calculated. Thus, we chose a sample size of 40 patients for each group.

Continuous data were presented as mean $\pm \mathrm{SD}$, and categorical data as number (\%). The results were tabulated, and statistical analysis was done using GraphPad InStat version 3.0. For categorical variables, chi-square test and Fishers exact test were applied. Continuous data was analysed using Two-sided unpaired Student's t-test. Repeated measures ANOVA was applied to see the trend of the 
haemodynamic parameters for the two groups and compare the two groups. A p value of $<0.05$ was considered to be statistically significant.

\section{Annexure 1}

Modified MPC Grading

In sitting position with mouth opened maximally, and without phonation.

Grade I: Soft palate, uvula, fauces, pillars visible.

Grade II: Soft palate, uvula, fauces visible.

Grade III: Soft palate, base of uvula visible.

Grade IV: Only hard palate visible.

IID

Distance from the lower border of the upper incisors to the upper border of the lower incisors, was measured using measuring tape, in sitting position.

\section{TMD}

Was measured using measuring tape in sitting position with neck extended, as the distance between the thyroid notch and symphysis menti with mouth closed.

\section{Modified Cormack-Lehane Grading}

- Grade 1: Full view of glottis.

- Grade 2a: Partial view of glottis.

- Grade 2b: Only posterior extremity of glottis or only arytenoid cartilages seen.

- Grade 3: Only epiglottis seen.

- Grade 4: Neither glottis nor epiglottis seen.

\begin{tabular}{|c|c|c|c|c|c|c|}
\hline \multicolumn{7}{|c|}{ RESULTS } \\
\hline \multicolumn{3}{|c|}{ Parameter } & \multicolumn{2}{|c|}{$\begin{array}{l}\text { Group A } \\
\text { (Mc Coy) }\end{array}$} & $\begin{array}{l}\text { Group B } \\
\text { (CTrach) }\end{array}$ & $\begin{array}{c}\mathbf{P} \\
\text { Value }\end{array}$ \\
\hline \multicolumn{3}{|c|}{ Age (yrs.) } & \multicolumn{2}{|c|}{$35.2 \pm 10.09$} & $35.9 \pm 9.58$ & 0.75 \\
\hline \multicolumn{3}{|c|}{ Gender (male: female) (\%) } & \multicolumn{2}{|c|}{$21: 19 ; 52: 48$} & $18: 22 ; 45: 55$ & 0.6 \\
\hline \multicolumn{3}{|c|}{ Height $(\mathrm{cm})$} & \multicolumn{2}{|c|}{$163.7 \pm 4.88$} & $163.3 \pm 6.04$ & 0.76 \\
\hline \multicolumn{3}{|c|}{ Weight (kg) } & \multicolumn{2}{|c|}{$63.9 \pm 6.97$} & $63.2 \pm 6.32$ & 0.63 \\
\hline \multicolumn{3}{|c|}{ ASA status I/II (\%) } & \multicolumn{2}{|c|}{$24 / 16 ;(60 / 40)$} & $22 / 18 ;(55 / 45)$ & 0.56 \\
\hline \multicolumn{3}{|c|}{$\operatorname{TMD}(\mathrm{cm})$} & \multicolumn{2}{|c|}{$7.81 \pm 0.5$} & $7.94 \pm 0.5$ & 0.23 \\
\hline \multirow{2}{*}{$\begin{array}{l}\text { IID } \\
(\mathrm{cm})\end{array}$} & \multirow{2}{*}{\multicolumn{2}{|c|}{$\begin{array}{l}\text { Without collar } \\
\text { With collar }\end{array}$}} & $4.87 \pm 0.53$ & \multirow[t]{2}{*}{0.0001} & $\begin{array}{ll}4.92 \pm 0.52 & 0.0001\end{array}$ & 0.70 \\
\hline & & & $4.19 \pm 0.45$ & & $4.29 \pm 0.45^{0.0001}$ & 0.33 \\
\hline \multirow{2}{*}{ MPC } & \multirow[b]{2}{*}{$\begin{array}{l}\text { With } \\
\text { collar }\end{array}$} & $\begin{array}{l}\text { I } \\
\text { II } \\
>\text { II }\end{array}$ & $\begin{array}{c}\overline{22} \\
18 \\
0\end{array}$ & \multirow{2}{*}{0.0001} & $\begin{array}{c}21 \\
19 \\
0\end{array}$ & 1 \\
\hline & & $\begin{array}{l}\text { I } \\
\text { II } \\
>\text { II }\end{array}$ & $\begin{array}{c}0 \\
19 \\
21\end{array}$ & & $\begin{array}{c}0 \\
20 \\
20\end{array}$ & 1 \\
\hline \multicolumn{7}{|c|}{$\begin{array}{c}\text { Table 1. Demographic Characteristics of Patients } \\
\text { in Group A and Group B }\end{array}$} \\
\hline \multicolumn{7}{|c|}{$\begin{array}{l}\text { Values are expressed as Mean } \pm \text { SD or number }(\%) \mathrm{SD}=\text { standard deviation, } \mathrm{ASA}= \\
\text { American society of anaesthesiologists, } \mathrm{TMD}=\text { thyromental distance, IID=inter- } \\
\text { incisor distance, MPC = Mallampati classification }\end{array}$} \\
\hline
\end{tabular}

The two groups were comparable in terms of the demographic factors viz. age, gender, height, weight, ASA status and thyromental distance. (table 1)

\section{Effect of Cervical Collar on Various Parameters in Both the Groups}

The MPC grade and inter-incisor distance worsened significantly after the application of cervical collar. However, both these parameters were comparable between the two groups before and after the application of cervical collar. (table 1).

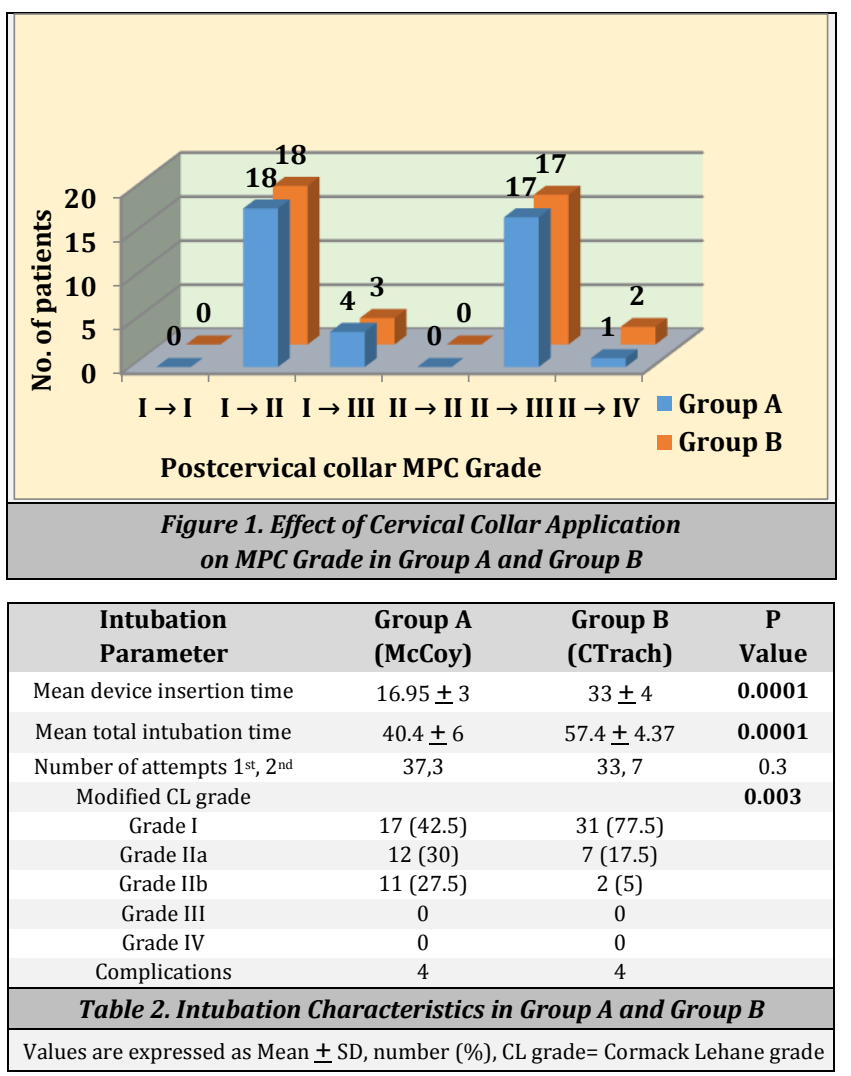
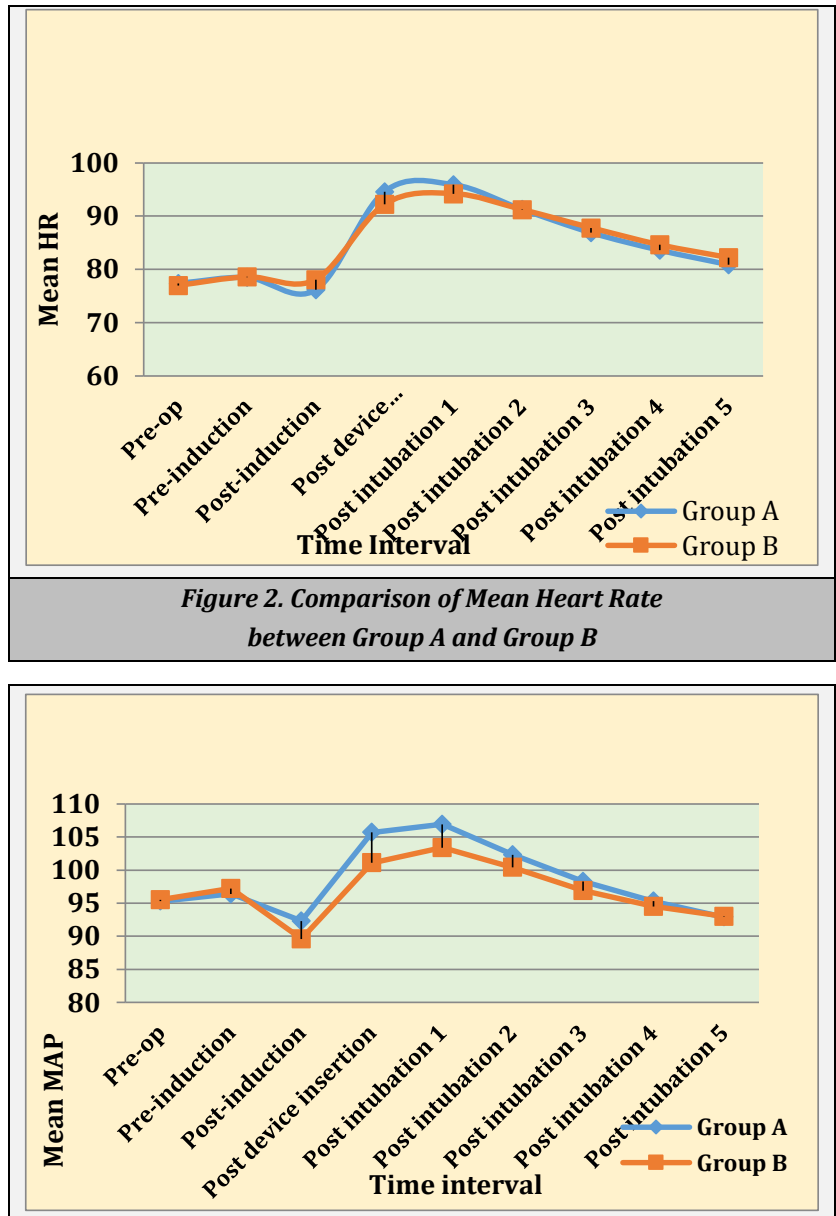

Figure 3. Comparison of Mean MAP between Group A and Group B 


\section{Intubation Characteristics of Both the Devices}

There was a significant difference in device insertion time, total intubation time and CL grade of both the groups. Number of attempts required, and airway complications encountered were comparable between the two groups. The intubation characteristics of both the devices have been summarized in Table 2.

The haemodynamic parameters recorded at various intervals were also comparable between the groups except for mean MAP value post device insertion and at 1 min post intubation was significantly higher in Group A.

\section{DISCUSSION}

Cervical spine motion restriction is imperative during intubation in trauma patients with suspected cervical spine injury. ${ }^{4}$ The use of cervical collar for the same, poses a significant hurdle as it makes conventional laryngoscopy difficult. ${ }^{6}$ The McCoy levering laryngoscope is a modified Macintosh laryngoscope, which has a hinged tip controlled by a lever on the handle which enables the epiglottis to be easily elevated without stressful force on the upper incisors or stressful neck extension.9,10 Since McCoy laryngoscope is easily available in Indian scenario and has been studied extensively in difficult airway patients, we have compared the use of this blade with a newer device, the LMA CTrach.

The LMA CTrach system is based on the Intubating Laryngeal Mask Airway (ILMA) with integrated fiberoptic channels and a detachable LCD screen. ${ }^{8}$ Thus, it overcomes the disadvantages of ILMA as it can be manipulated and aligned with the larynx under vision. ILMA (Fastrach) is extensively used in emergency and difficult intubation settings ${ }^{11}$ but has higher failure rates in anteriorly placed larynx and due to folding of epiglottis making endotracheal tube placement difficult. ${ }^{12}$ Moreover, esophageal intubation and accidental extubation during removing ILMA are also common. ${ }^{13,14}$ In a study by S Saini, R Bala and R Singh evaluating the use of Intubating Laryngeal Mask Airway (ILMA) as an intubation conduit in patients with cervical collar, the overall success rate was only $85.7 \%$ with failed intubation in 4 out of 35 patients. ${ }^{14}$ In a study by Liu et al. comparing the success of intubation between Fastrach and LMA CTrach, the number of 1 st $/ 2^{\text {nd }} / 3^{\text {rd }} /$ failed attempts were $93 / 29 / 10 / 5$ and $125 / 7 / 2 / 0$ respectively with less median number of attempts with LMA CTrach. ${ }^{12}$ The CTrach has an epiglottis elevating bar, which elevates the epiglottis during passage of the endotracheal tube (ETT) through the CTrach into the larynx. This bar has an aperture through which the anatomy anterior to the bar is viewed. The CTrach's shape is based on magnetic resonance imaging of the human airway, to enable a close fit with the oropharyngeal curve and optimal alignment with the laryngeal inlet. ${ }^{15}$

In our study the presence of cervical collar worsened the airway parameters viz. mouth opening, inter-incisor distance and Mallampati class. Thus, this simulation exercise proved that cervical collar significantly impairs the airway rendering it difficult, as has been shown in various other studies. ${ }^{13,16}$ The insertion of laryngoscopy blade/LMA requires a mouth opening of $>2 \mathrm{~cm}$ and so was not a problem in our study. ${ }^{17}$
The device insertion time and total intubation time was lesser with McCoy compared to LMA CTrach as attaching the viewer after inserting the LMA and maneuvering to obtain a good glottic exposure takes some time. Though time taken is more with CTrach, continuous ventilation through LMA is possible throughout which is an added advantage. In a study by Jain et al. the device insertion time with McCoy laryngoscopy was 14 sec and total intubation time was 26 sec. ${ }^{18} \mathrm{Z}$. I. Arsalan et al. compared Airtraq and LMA CTrach devices for tracheal intubation in patients with rigid collar and found the time to view the glottis to be $37.6+16.7 \mathrm{sec}$ with LMA CTrach and total intubation time was $66.3 \mathrm{sec}$ which is similar to our findings. ${ }^{19}$

Despite being time consuming, the glottic view obtained as judged by the modified CL grade, was better with LMA CTrach than McCoy blade. The incidence of CL grade I was more in Group B (77.5\%) than Group A (42.5\%). None of the patients had CL grade III/IV views in either group. R Komatsu et al. used Mc Coy laryngoscope to intubate patients with rigid cervical collar and found CL grade IIa, IIb to be more common in McCoy configuration. ${ }^{16}$ Till date there have been no studies that comment on CL grading in patients intubated using LMA CTrach in presence of cervical collar. Tripathi et al. studied the use of CTrach in patients with rigid cervical collar and commented on the POGO score to be $75-100 \% / 50$ $74 \% /<50 \%-22 / 8 / 0$ with $100 \%$ success rate. ${ }^{20}$ However, POGO score is more subjective compared to CL grade with less intra-rater reliability whereas CL grade is universally used and is more popular amongst anesthesiologists with good reproducibility. Our study has been able to present this valuable data regarding LMA CTrach in patients with cervical collar which has been missed out in other studies of similar kind. Data regarding CL grade in patients intubated with LMA CTrach is available in patients with normal airway and suggest the preponderance of CL grade I and II (I>II) at the time of intubation. ${ }^{15}$ Sometimes maneuvering may be required to obtain the best glottic view which we also encountered during our study. Timmerman et al. in his study showed improvement in CL grades with maneuvers in patients with CL grade II and above. ${ }^{21}$

The haemodynamic pressor response to laryngoscopy is lesser with McCoy and LMA CTrach compared to Macintosh laryngoscope.22,23,24 There was significant increase in heart rate and MAP after device insertion and intubation in both the groups, with the MAP slightly more in McCoy group than CTrach group. Intubation using CTrach generates low sympathetic stress response because the proprioceptors at the base of the tongue are not stimulated.

\section{Limitations}

A limitation of our study was that the anaesthetist recording the laryngoscopic view could not be blinded to the device being used, so the observer's bias could not be eliminated. We have used Cormack and Lehane grading system which is applicable to grade the laryngoscopic view during direct laryngoscopy. Currently, there is no definitive accepted grading system for video laryngoscopes; therefore, in our study, we have used the CL grading. The study was conducted in patients with normal airways and the same results may not be reproduced in patients who have actual cervical spine pathology. 


\section{CONCLUSIONS}

Application of cervical collar significantly impairs the airway. Both McCoy laryngoscope and LMA CTrach are comparable in terms of success rate, number of attempts required, and associated complications, however, although McCoy laryngoscope takes lesser time for intubation, the glottic view is definitely better with CTrach and the pressor response also seems to be slightly lesser which is an added advantage. Thus, both McCoy laryngoscope and LMA CTrach can be used as suitable alternatives to conventional laryngoscopy, as they are known to improve glottic exposure and have proved to be an effective aid in difficult intubation scenario, as is the case with presence of cervical collar.

\section{ACKNOWLEDGEMENT}

We thank Dr. Sunil Kant Kamra, Ex Head of Department of Anaesthesia, JLN Hospital and Research Centre, Bhilai, for his valuable guidance.

\section{REFERENCES}

[1] Grossman MD, Reilly PM, Gillet T, et al. National Survey of the Incidence of Cervical Spine Injury and Approach to Cervical Spine Clearance in US trauma centre. J Trauma 1999;47(4):684-90.

[2] Holly LT, Kelly DF, Counelis GJ, et al. Cervical spine trauma associated with moderate and severe head injury: incidence, risk factors and injury characteristics. J Neurosurg 2002;96(Suppl 3):285-91.

[3] Hastings RH, Kelly SD. Neurologic deterioration associated with airway management in a cervical spine injured patient. Anesthesiology 1993;78(3):580-3.

[4] Henry S, Brasel K, Stewart R. Advanced trauma life: support student course manual. Chap - 1. Initial Assessment and Management. 10 $0^{\text {th }}$ edn. Chicago: American College of Surgeons 2018: p. 4-20.

[5] Goutcher CM, Lochhead V. Reduction in mouth opening with semi-rigid cervical collars. British Journal of Anaesthesia 2005;95(3):344-8.

[6] Smith CE, Pinchak AB, Sidhu TS, et al. Evaluation of tracheal intubation difficulty in patients with cervical spine immobilization: fiberoptic (WuScope) versus conventional laryngoscopy. Anaesthesiology 1999;91(5):1253-9.

[7] Crosby ET, Lui A. The adult cervical spine: implications for airway management. Can J Anaesth 1990;37(1):7793.

[8] LMA-CTrach ${ }^{\mathrm{TM}}$ instruction manual. The Laryngeal Mask Company Ltd., 2006: p. 1-22.

[9] Gabbott DA. Laryngoscopy using the McCoy laryngoscope after application of a cervical collar. Anaesthesia 1996;51(9):812-4.
[10] Uchida T, Hikawa Y, Saito Y, et al. The McCoy levering laryngoscope in patients with limited neck extension. Can J Anaesth 1997;44(6):674-6.

[11] Ferson DZ, Rosenblatt WH, Johansen MJ, et al. Use of the intubating LMA-Fastrach ${ }^{\text {TM }}$ in 254 patients with difficultto-manage airways. Anesthesiology 2001;95(5):1175-81.

[12] Liu EH, Goy RW, Lim Y, et al. Success of tracheal intubation with intubating laryngeal mask airways: a randomized trial of the LMA Fastrach ${ }^{\text {TM }}$ and LMA CTrach $^{\text {TM. }}$. Anesthesiology 2008;108(4):621-6.

[13] Komatsu R, Nagata O, Kamata $K$, et al. Intubating laryngeal mask airway allows tracheal intubation when the cervical spine is immobilized by a rigid collar. $\mathrm{Br} \mathrm{J}$ Anaesth 2004;93(5):655-9.

[14] Saini S, Bala R, Singh R. Evaluation of the Intubating Laryngeal Mask Airway (ILMA) as an intubation conduit in patients with a cervical collar simulating fixed cervical spine. South Afr J Anaesth Analg 2017;23(2):40-4.

[15] Liu EHC, Goy RWL, Chen FG. The LMA CTrach, a new laryngeal mask airway for endotracheal intubation under vision: evaluation in 100 patients. $\mathrm{Br} J$ Anaesth 2006;96(3):396-400.

[16] Komatsu R, Kamata K, Sessler DI, et al. A comparison of the Airway Scope and McCoy laryngoscope in patients with simulated restricted neck mobility. Anaesthesia 2010;65(6):564-8

[17] Brain AI, Verghese C, Addy EV, et al. The intubating laryngeal mask. I. Development of a new device for intubation of the trachea. Br J Anaesth 1997;79(6):699703.

[18] Jain D, Bala I, Gandhi K. Comparative effectiveness of McCoy laryngoscope and CMAC (®) videolaryngoscope in simulated cervical spine injuries. J Anaesthesiol Clin Pharmacol 2016;32(1):59-64

[19] Arslan ZI, Yildiz T, Baykara ZN, et al. Tracheal intubation in patients with rigid collar immobilisation of the cervical spine: a comparison of Airtraq and LMA CTrach devices. Anaesthesia 2009;64(12):1332-6.

[20] Tripathi DC, Jha PS, Trivedi LP, et al. LMA CTrach aided endotracheal intubation in simulated cases of cervical spine injury: a series of 30 cases. Saudi J Anaesth 2013;7(2):165-9.

[21] Timmermann A, Russo S, Graf BM. Evaluation of the $\mathrm{CTrach}^{\mathrm{TM}}$-an intubating LMA with integrated fibreoptic system. British Journal of Anaesthesia 2006;96(4):51621.

[22] Haidry MA, Khan FA. Comparison of haemodynamic response to tracheal intubation with Macintosh and McCoy laryngoscopes. J Anaesthesiol Clin Pharmacol 2013;29(2):196-9.

[23] Bilgin H, Bozkurt M. Tracheal intubation using the ILMA, CTrach or McCoy laryngoscope in patients with simulated cervical spine injury. Anaesthesia 2006;61(7):685-91.

[24] Sarkar J, Anand T, Kamra SK. Haemodynamic response to endotracheal intubation using CTrach assembly and direct laryngoscopy. Saudi J Anaesth 2015;9(4):343-7. 